\title{
Social communication, executive functions and expressive language in children with ASD with ADHD symptoms - an exploratory study
}

\author{
CRISTINA BALAS-BACONSCHI ${ }^{1}$ \\ CARMEN POP ${ }^{2}$ \\ CRISTINA COSTESCU ${ }^{3}$
}

\begin{abstract}
There are several factors associated with language deficits in children with autism spectrum disorder (ASD). Previous research identified theory of mind difficulties, executive function deficits, abstract thinking and social information processing deficits as being linked with language deficits in ASD. However when it comes to the co-occurance of ASD and ADHD symptoms studies have different theories regarding the way language and social communication deficits emerge. In our exploratory study we propose to investigate the role of the auditory memory in the development of vocabulary and his indirect relation to social interaction and communication. In order to reach our goals we have described 6 case studies of children with ASD and ADHD symptoms aged 7 years old. Our results reveal that even if the participants in this study have a low to medium performance in the auditory memory task, this does not seem to impact expressive language to a high extend. Moreover the majority of the participants in our study had good vocabulary skills but seem to have difficulties in social communication. This study was designed to be a preliminary investigation of the connection between different factors that may influence social communication in children with ASD and ADHD symptoms and it can bring new research directions in the domain.
\end{abstract}

\section{Keywords}

autism spectrum disorder, language development, auditory memory, social communication

\footnotetext{
${ }^{1}$ Cristina Balas-Baconschi, Special Education Department, Faculty of Psychology and Educational Sciences, Babes-Bolyai University;

${ }^{2}$ Carmen Pop, Special Education Department, Faculty of Psychology and Educational Sciences, BabesBolyai University;

${ }^{3}$ Cristina Costescu, Special Education Department, Faculty of Psychology and Educational Sciences, Babes -Bolyai University.

Correspondence concerning this article should be addressed to: Cristina Costescu, Special Education Department, Faculty of Psychology and Educational Sciences, Babes -

Bolyai University, No.7, Sindicat elor Street 400015, Cluj-Napoca, Cluj, Romania.

E-mail: christina.costescu@gmail.com
} 